Seitenfläche entsprechen sơlte, müssen wir beim Südpole suchen, also in Mare australe. Die Mitte dieses 》Meeres « fällt nicht genau mit dem Südpole zusammen; in $\lambda 195^{\circ}$ reicht Mare australe nach Antoniadis Karte nur bis $-75^{\circ}$, in $\lambda$ I $5^{\circ}$ dagegen bis $-50^{\circ}$, sodaß die Mitte in $\lambda=15^{\circ}$, $\beta=-78^{\circ}$ liegt, also in unmittelbarer Nähe der Mitte des südlichen Polarflecks. Auf dem diametral entgegengesetzten Punkte der Oberfläche liegt der Schneefleck Olympia. Die Größe der vier Einsenkungen scheint ungefähr von derselben Ordnung zu sein: Mare Acidalium ist etwa $30^{\circ}-35^{\circ}$ groß, Propontis $35^{\circ}-40^{\circ}$, Utopia $40^{\circ}-50^{\circ}$ und Mare australe $55^{\circ}$, also im Mittel etwa $40^{\circ}$.

Die Tiefe der vier Einsenkungen ist vermutlich, wie bei unserer Erde, gering im Vergleiche mit der Abplattung, aber sie sind nichtsdestoweniger von der größten Bedeutung für die physischen Verhältnisse des Planeten. Wenn der nördliche Polarfleck auf einer Hochebene liegt, so muß das Schnee wasser sich in den drei Einsenkungen der nördlichen Halbkugel sammeln, weil sie von der südlichen Einsenkung durch höhere Gebiete getrennt sind; umgekehrt muß das Wasser vom Südpole in der antarktischen Niederung verbleiben. So schematisch sind die tatsächlichen Verhältnisse auf Mars natürlich nicht; die »Wälle "zwischen den polaren Morasten auf der einen Seite und der südlichen Einsenkung auf der anderen sind von großen Spalten durchbrochen, aber die Auflösung des nördlichen Schneeflecks hat tatsächlich nur einen sehr geringen Einfluß auf das Aussehen der südlichen Meere, wie später auseinandergesetzt wird. Wenn die höheren Gebiete zwischen den Morasten und den südlichen Meeren beim Zusammenziehen des Planeten geborsten sind, so liegt es nahe anzunehmen, daß die Bruchlinien in allen drei Fällen dieselbe Anordnung zeigen werden. Die Moraste sind ferner unter sich durch große Kanäle verbunden, nämlich PieriusCallirrhoe, Tanais-Eurotas und Gyndes-Heliconius.

Wenn die besondere Form der Einsenkungen durch die Art und Weise, in welcher sich der Planet zusammengezogen hat, bedingt wäre, so müßten ähnliche Einsenkungen auch auf anderen relativ alten Himmelskörpern vorhanden sein. Es läßt sich in der Tat auf dem Monde eine Analogie zum Acidalium-System nachweisen, nämlich die "Meere" am rechten Mondrande. Mare Serenitatis entspricht Acidalium, Mare Tranquillitatis Niliacus Lacus, während die beiden "Mondkanäle" Mare Nectaris und Mare Foecunditatis - die dem bloßen Auge als breite Streifen erscheinen - Jamuna und Hydaspes ersetzen. An die Stelle des Achilles Pons treten auf dem Monde die Vorgebirge bei Plinius, ja, selbst Palus Acidalium hat eine Analogie in Mare Frigoris. Die Ähnlichkeit mag natürlich auf einer Anhäufung von Zufälligkeiten beruhen, aber die Übereinstimmung ist immerhin groß genug, um hier hervorgehoben zu werden.

Während die vier Einsenkungen der Marsoberfläche durch ihre dunkle Farbe im Frühjahre deutlich gekennzeichnet sind, ist es kaum möglich, die hypothetischen Hochebenen an den Spitzen des Tetraeders nachzuweisen. Da die höheren Gebiete zwischen den Morasten, wie Tempe-Arcadia, Elysium und Isidis, selbst im Sommer glänzend weiß sein können, so liegt es nahe, ähnliche Eigenschaften bei den Hochebenen anzunehmen, und die "Spitzen" des Tetraeders sind daher vielleicht in Aeria-Edom, Tharsis-Thaumasia-Menınonia und Aeolis oder Eridania zu suchen. In Aeria, Edom und Memnonia treten häufig helle, veränderliche Massen auf, die schon Dawes für Wolkenmassen hielt, welche sich auf den Südseiten von großen Hochebenen kondensieren. Wenn. wir die hypothetischen Hochländer in Memnonia und Erïdania annehmen, so würden die großen Binnenmeere eine gewaltige Kluft zwischen den mutmaßlichen Hochländern darstellen. Aber wir besitzen keine Mittel, diese Konsequenzen der Theorie zu prüfen, und wir wollen uns daher mit der Folgerung begnügen, daß die Lage der vier Einsenkungen in den Hauptzügen mit der Tetraedertheorie übereinstimmt.

Hörsholm, I 9 I 6 September.

$$
\text { H. E. Lau. }
$$

\title{
Sonnenfinsternis 1917 Januar 22.
}

Das Ende der partiellen Sonnenfinsternis am 22. Januar I9 7 war hier bei klarem Himmel ziemlich gut zu beobachten. Die Sonne stand allerdings noch dicht über Häusern, und der Rand war sehr wallend. Am 6-zöll. Refraktor sah ich im projizierten Bilde um $20^{\mathrm{h}} 5^{6^{\mathrm{m}}} 5^{\mathrm{s}} \cdot 2 \mathrm{M}$. E. Z. den Eingriff des Mondrandes noch eben deutlich, um $20^{\mathrm{h}} 56^{\mathrm{m}} 10^{\mathrm{s}} 2$ war er verschwunden.

Bonn, I 9 I 7 Januar 30.

\section{F. Küstner.}

\section{Notiz zur Osterformel. Von $\mathcal{F}$. Hartmann.}

Die in Nr. 4876 p. 62 aufgestellte Behauptung, daß die von mir in A. N. 4473 (187. I 29 , r 9 I I) veröffentlichte Osterformel Herrn $A$. Wedemeyer schon im Jahre 1862 bekannt gewesen sei, zwingt mich zu einer kurzen Entgegnung. Daß die Priorität der Aufstellung meiner Formel nachträglich von verschiedenen Seiten in Anspruch genommen wurde, kann dieser Formel nur zur Ehre gereichen. Wird dadurch doch ihre bisher unübertroffene Einfachheit immer wieder anerkannt. Aber ebensowenig wie die früheren Einwände (vergl. A. N. $454 \mathrm{I}$ und 4560) ist der jetzt in Rede stehende Anspruch begründet. Herr Wedemeyer hat, was mir bei der Ableitung meiner Formel nicht bekannt war, im Jahre I 862 im $\gg \mathrm{Han}$ - noverschen Volksschulboten " eine gute Osterformel veröffentlicht, die der meinigen $z$ war etwas ähnlich, mit ihr aber noch lange nicht identisch ist. Daß zwischen den verschiedenen Osterformeln stets nahe Beziehungen bestehen, daß sie sich in manchen Teilen sogar gleichen müssen, liegt ja in der Natur der Sache. Aber die Wedemeyersche Formel unterscheidet sich in zwei Punkten sehr zu ihrem Nachteil von der meinigen. Erstens ist es zur Anwendung jener Formel, genau wie bei der Gaußschen, notwendig, sich für jedes Jahrhundert $z$ wei Konstanten zu merken, während in meiner Formel nur die eine neue Konstante $M$ auftritt, die andere dagegen durch die Jedem schon bekannte Differenz $D$ zwischen dem gre- 